\title{
Metathetical Redox Reaction of (Diacetoxyiodo)arenes and Iodoarenes
}

\author{
Antoine Jobin-Des Lauriers and Claude Y. Legault * \\ Received: 25 November 2015 ; Accepted: 15 December 2015 ; Published: 17 December 2015 \\ Academic Editors: Wesley Moran and Arantxa Rodríguez \\ Centre in Green Chemistry and Catalysis, Department of Chemistry, University of Sherbrooke, \\ 2500 boul. de l'Université, Sherbrooke, QC J1K 2R1, Canada; Antoine.Jobin-Des.Lauriers@USherbrooke.ca \\ * Correspondence: claude.legault@usherbrooke.ca; Tel./Fax: +1-819-821-8017
}

\begin{abstract}
The oxidation of iodoarenes is central to the field of hypervalent iodine chemistry. It was found that the metathetical redox reaction between (diacetoxyiodo)arenes and iodoarenes is possible in the presence of a catalytic amount of Lewis acid. This discovery opens a new strategy to access (diacetoxyiodo)arenes. A computational study is provided to rationalize the results observed.
\end{abstract}

Keywords: hypervalent iodine; (diacetoxyiodo)arenes; metathesis

\section{Introduction}

In the recent years, the development of hypervalent iodine-mediated synthetic transformations has been receiving growing attention [1-5]. This is not surprising considering the importance of improving oxidative reactions and reducing their environmental impact. Hypervalent iodine reagents are a great alternative to toxic heavy metals often used to effect similar transformations [6-10]. Among the plethora of iodine(III) compounds [11], it is undeniable that the (diacetoxyiodo)arenes remain the most common and used ones [12]. They are usually stable solids and can serve as precursors to numerous other iodanes, such as other (diacyloxyiodo)arenes and [hydroxy(tosyloxy) iodo]arenes [13,14]. Access to (diacetoxyiodo)arenes is, of course, usually accomplished by the oxidation of the corresponding iodoarene substrate. The traditional method involves peracetic acid oxidation in acetic acid [15]. One unexploited strategy to access these specific reagents is the metathetical redox reaction between (diacetoxyiodo)arenes and related iodoarenes (Scheme 1a).

While it is widely accepted that the ligands on iodane species are typically easily exchanged, the interconversion of oxidation states between $\lambda^{3}$-iodane and iodoarenes is much rarer. The first example of such a metathetical redox reaction was reported by Koser et al., using [hydroxy(tosyloxy)-iodo] benzene (HTIB) as the oxidant, enabling transfer to a variety of iodoarenes (Scheme 1b) [16]. Two applications of this initial method were subsequently reported, including one example using [bis(trifluoroacetoxy)iodo]benzene (BTI) $[17,18]$. More recently, Ciufolini et al. have reported the iodonium salt metathesis reaction with iodoarenes (Scheme 1c) [19].

To the best of our knowledge, the metathetical redox reaction between (diacetoxyiodo)arenes and iodoarenes has never been reported. Expanding the scope of iodane metathesis to these commonly used reagents would be of great interest as an alternative synthetic method. Herein we report a protocol to achieve such a metathesis of (diacetoxyiodo)benzene and iodoarenes. We also provide a computational study to determine the origin of the observed reactivity. 


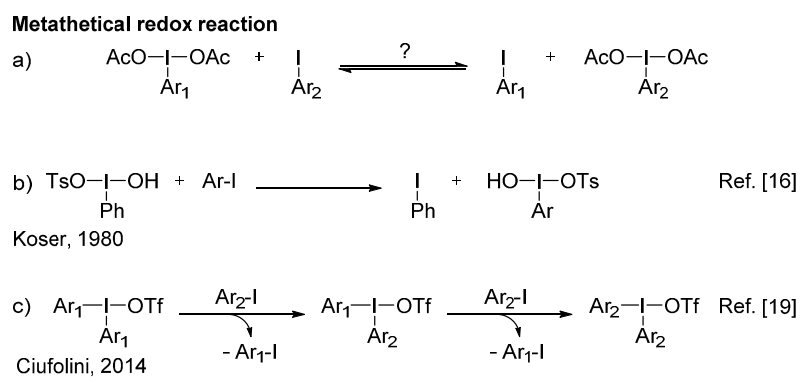

Scheme 1. Concept and examples of metathetical redox reactions of iodanes and iodoarenes.

\section{Results and Discussion}

The reactivity of HTIB and (diacetoxyiodo)benzene (DIB) toward iodoarenes was evaluated, using conditions analogous to Koser's successful iodane metathesis with HTIB [16]. We submitted $p$-methyliodobenzene and $p$-bromoiodobenzene to iodane metathesis with HTIB (Scheme 2a) and DIB at room temperature (Scheme $2 b$ ). In accordance with the results reported by Koser, equilibrium is achieved for the reaction with HTIB within $96 \mathrm{~h}$. In contrast, no metathesis was observed after $72 \mathrm{~h}$ with DIB.

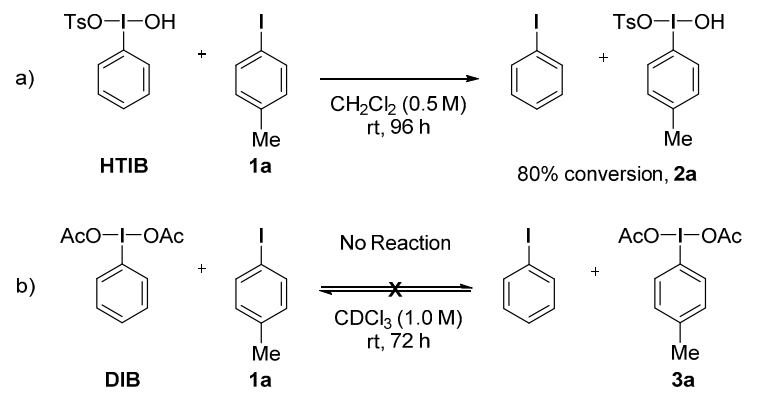

Scheme 2. Evaluation of the reactivity of DIB toward the metathetical redox process.

These results demonstrate the very different reactivity profiles of DIB and HTIB. The origin of this reactivity dichotomy was investigated. In a recent computational study, we have suggested that reaction with HTIB would involve, at room temperature, iodonium intermediates, through a ligand dissociation mechanism [20]. Due to the large difference in effective electronegativity between the hydroxy and tosyloxy groups on HTIB, ionization in solution is achievable at room temperature [21,22]. In contrast, (diacetoxyiodo)arenes have identical ligands involved in the three-center four-electron bonding, and thus dissociation is expected to be difficult [23]. We have evaluated the energetic of dissociation for HTIB, DIB, and diphenyliodonium salt 4 (see the computational details in Section 4), the results are illustrated in Scheme 3.

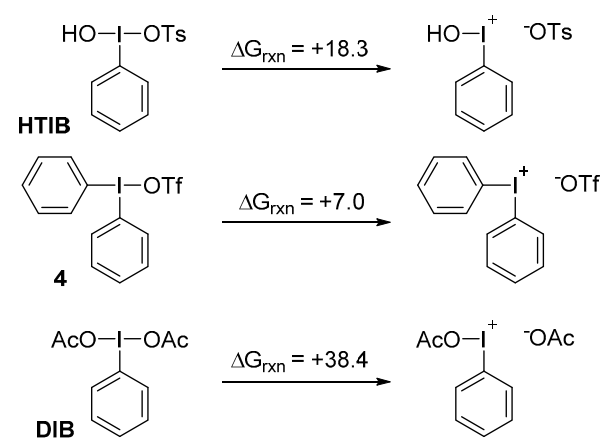

Scheme 3. Energetics of ligand dissociation on DIB, 4, and HTIB (energies reported in $\mathrm{kcal} / \mathrm{mol}$ ). 
As suspected, the dissociation of the tosyloxy group is calculated to be endergonic, but feasible. Dissociation of the triflate anion in the diphenyliodonium salt $\mathbf{4}$ is predicted to be even more facile at room temperature. In contrast, the calculations suggest that dissociation of an acetoxy group on DIB at room temperature would be too difficult.

The dissociation energies seem to be correlated to the intrinsic reactivity of these iodanes toward metathesis. We postulated that the key to the metathetical redox process might occur by the formation of an iodonium intermediate. If it is the case, this could be achieved with DIB by promoting the dissociation of an acetoxy group through the use of a Lewis acid. We elected to use $\mathrm{BF}_{3} \cdot \mathrm{OEt}_{2}$, as it was shown in numerous methodologies to be a proficient and compatible Lewis acid with DIB $[24,25]$. DFT Calculations, using $\mathrm{BF}_{3} \cdot \mathrm{OMe}_{2}$ as a model, suggest that this Lewis acid would permit the formation of an iodonium intermediate at room temperature, as illustrated in Scheme 4.

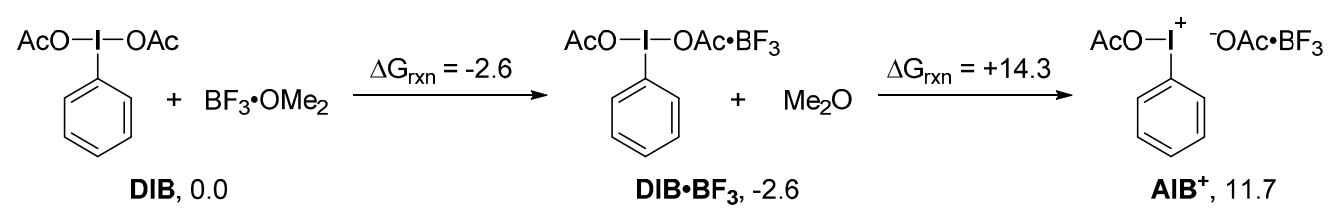

Scheme 4. Energetics of ligand dissociation on $\mathrm{DIB}$ with $\mathrm{BF}_{3} \cdot \mathrm{OMe}_{2}$ (energies reported in $\mathrm{kcal} / \mathrm{mol}$ ).

With this in mind, we tested the iodane metathesis between DIB and $p$-bromoiodobenzene (1b) in the presence of $5 \mathrm{~mol} \%$ of $\mathrm{BF}_{3} \cdot \mathrm{OEt}_{2}$ (Equation (1)). To our delight, the metathetical redox process proceeded cleanly to attain equilibrium within $67 \mathrm{~h}$. The calculated free energy of reaction was predicted to be $+0.5 \mathrm{kcal} / \mathrm{mol}$, which is in good agreement with the experimentally measured value of $+0.24 \mathrm{kcal} / \mathrm{mol}$. The reaction did also proceed with $p$-methyliodobenzene (1a), but formation of a suspension pointed to a Friedel-Crafts side reaction of 1a with activated DIB, and was not investigated further.
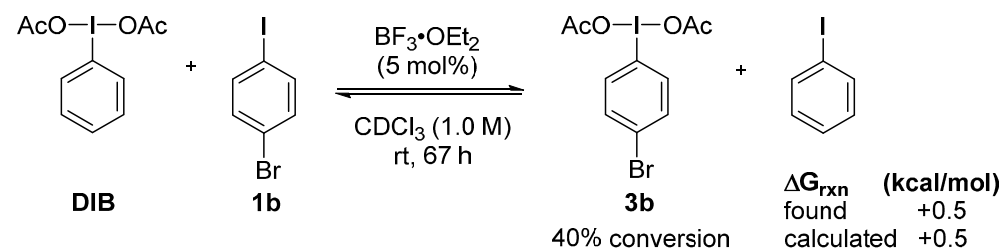

With these promising results in hand, the reaction mechanism was investigated using DFT calculations (see computational details in Section 4). A single electron transfer (SET) redox process between the iodonium intermediate and the iodoarene was considered (Equation (2)). The transfer was found to be energetically too costly to occur readily at room temperature. In accordance with the calculations, it was found that light had no effect on the rate of reaction in the conditions described in Equation (1), further suggesting that the process does not involve a SET.

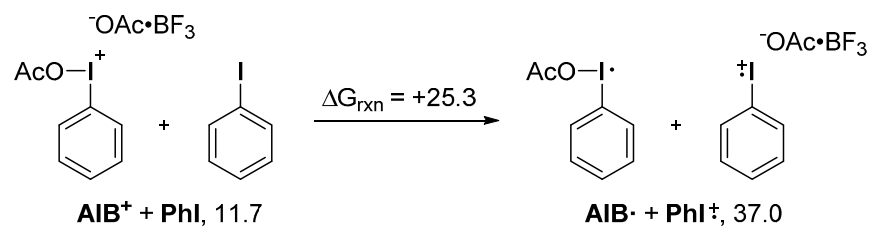

An ionic mechanism was thus considered. The intermediates evaluated are illustrated in Scheme 5. This mechanism has similarities to the one proposed by Ciufolini $e$ al. for diaryliodonium salt metathesis [19], with the exception of the transferred group being easily dissociated. For the sake of simplicity, the aryl (Ar) group in the intermediates is a simple phenyl group and the initial iodane is 
DIB. The transition states leading to the different intermediates described were not studied, as it was assumed that these processes of association and dissociation do not require large activation energies.

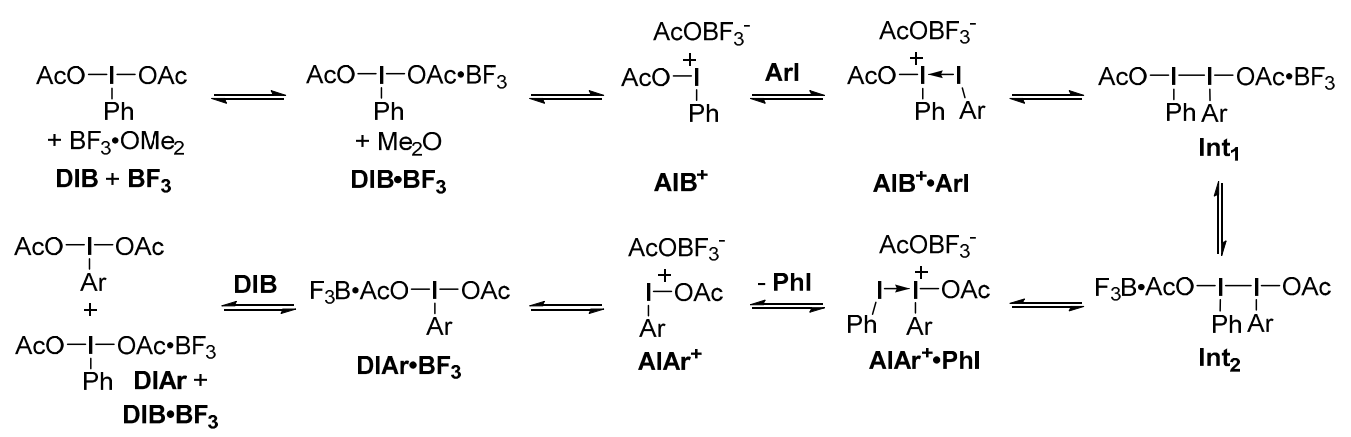

Scheme 5. Reaction mechanism evaluated using DFT calculations (Ar $=\mathrm{Ph}$ for simplicity).

The energetic properties of all these intermediates were calculated and are illustrated in an energy diagram (Scheme 6). As described in Scheme 6, formation of intermediate AIB $^{+}$, following complexation by $\mathrm{BF}_{3} \cdot \mathrm{OMe}_{2}$, was found to be an endergonic but feasible process at room temperature. At this point, the iodonium intermediate can act as a Lewis acid toward an iodoarene (in this case iodobenzene) to form adduct $\mathrm{AIB}^{+}$. ArI. Association of the $\mathrm{AcOBF}_{3}{ }^{-}$counterion led to Int $\mathbf{I}_{\mathbf{1}}$. By internal Lewis acid migration, Int $_{2}$ would be accessed. This intermediate can then lead to $\mathrm{AIAr}^{+}$. PhI adduct, which can liberate iodobenzene and finally provide, by transfer of $\mathrm{BF}_{3}$ to another DIB molecule, the newly formed (diacetoxyiodo)arene (DIAr). The calculations thus suggest an ionic mechanism relying on the asymmetry or activation of the iodane moiety to initiate the metathetical redox process, through a key iodonium intermediate.

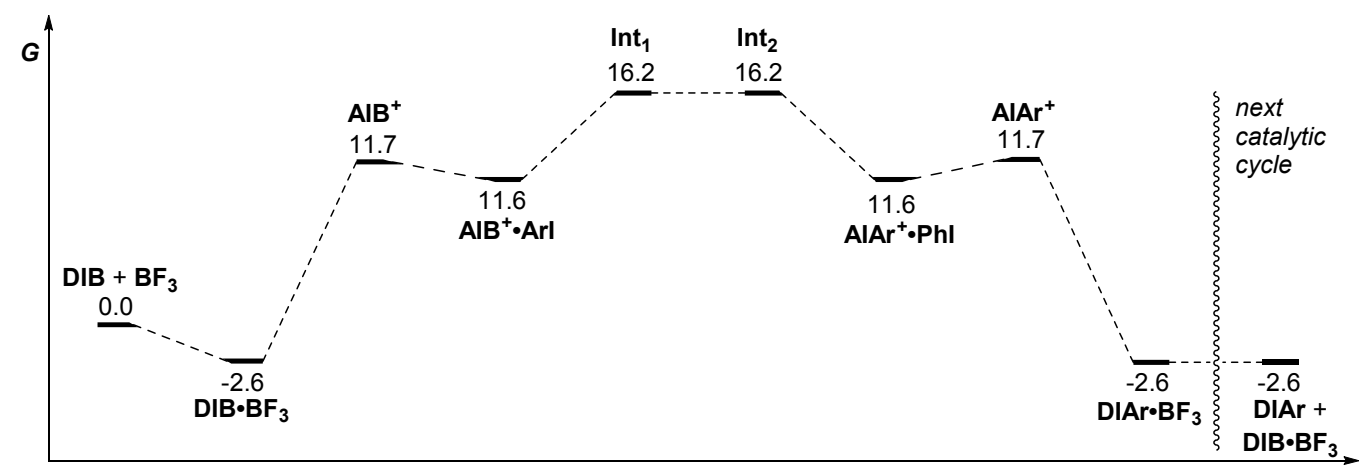

Scheme 6. Energy diagram of the proposed mechanism (energies reported in $\mathrm{kcal} / \mathrm{mol}$ ).

Passage through a non-symmetric iodane intermediate could explain the metathesis reported by Koser et al., in which iodoarene 5 is converted to its corresponding cyclic iodane 6 by the action of BTI, without the need of a catalyst (Equation (3)) [17].

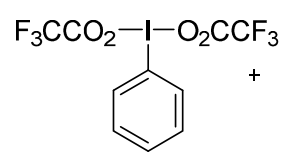

BTI<smiles>CC(O)(c1ccccc1)c1ccccc1I</smiles>

5

ge woul

$\overrightarrow{\mathrm{CH}_{2} \mathrm{Cl}_{2}, \mathrm{rt}, 4.5 \mathrm{~h}}$

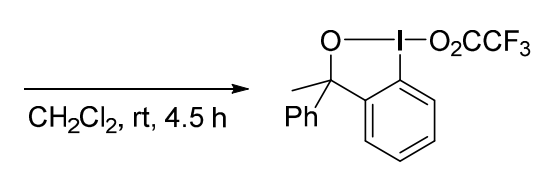

$6,50 \%$

In this reaction, rapid ligand exchange would lead to intermediate Int-A (Scheme 7). Due to the large difference in effective electronegativity between the alkoxy and trifluoroacetoxy groups, dissociation of the latter to furnish the iodonium intermediate Int-B is conceivable. Furthermore, 
the fact that Int-B binds together both $\mathrm{I}(\mathrm{I})$ and I(III) partners might facilitate the metathetical redox process.

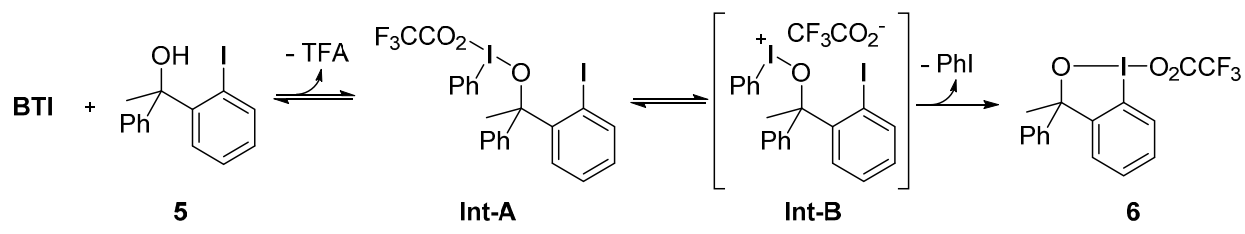

Scheme 7. Proposed intermediate to rationalize reactivity of BTI toward 5.

Calculations using $t-\mathrm{BuOH}$ as a model alcohol support this proposal, as described by the energetic values illustrated in Scheme 8. While dissociation of a trifluoroacetate group on the symmetric BTI was calculated to be too costly to occur at room temperature. In contrast, due to the lower effective electronegativity of the $t$-butoxy compared to the trifluoroacetoxy group, the dissociation on the non-symmetric tBTFIB is facilitated.

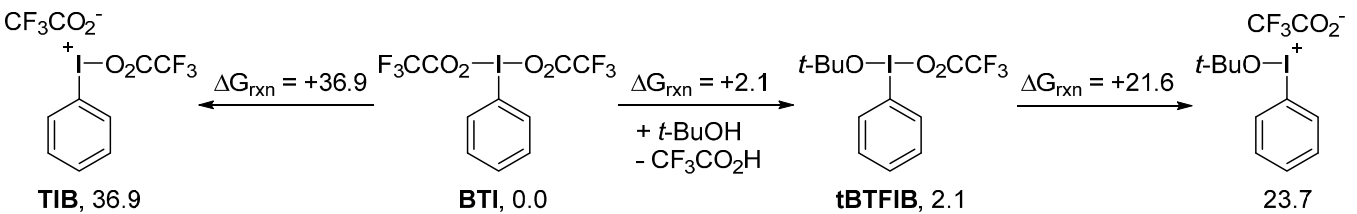

Scheme 8. Alcohol-promoted formation of an iodonium intermediate.

This alternative activation mechanism raises an important point concerning the presence of water in these reactions. As a protic solvent similar to an alcohol, water might act as an activator to promote acyloxy group dissociation. The energetic values of water exchange and possible acyloxy dissociation were computed for both DIB and BTI; the results are illustrated in Scheme 9. In the case of DIB, even the intermediate species [hydroxy(acetyloxy)iodo]benzene (HAIB) would not permit dissociation of the acetyloxy group to form the iodonium intermediate at room temperature. In contrast, the calculations suggest that dissociation of the trifluoroacetoxy group on the analogous [hydroxy(trifluoroacetoxy)iodo]benzene (HTFIB) intermediate might be possible at room temperature. In essence, even if BTI is found to be more stable than HTFIB, the latter would possess reactivity similar to Koser's reagent (HTIB). The main difference between DIB, BTI, and HTIB can simply be explained by the better nucleofugality of $\mathrm{TsO}^{-}$and $\mathrm{CF}_{3} \mathrm{CO}_{2}^{-}$vs. $\mathrm{AcO}^{-}$.

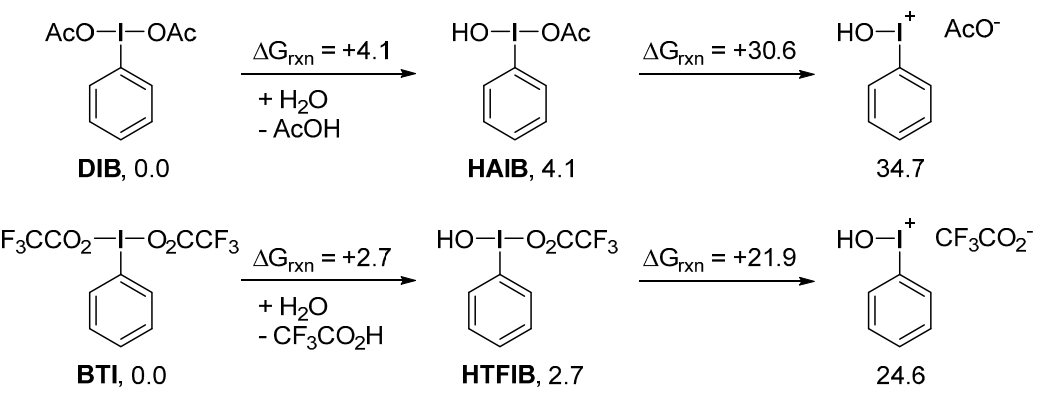

Scheme 9. Water-promoted formation of iodonium intermediates.

These calculations support the non-reactivity observed for DIB even in non-anhydrous solvents. In contrast, we could expect reactivity from BTI even at room temperature under non-anhydrous conditions. To test this hypothesis, BTI was let to react with $p$-bromoiodobenzene at room temperature without strict exclusion of water. For comparison, a similar experiment involving $5 \mathrm{~mol} \%$ of $\mathrm{BF}_{3} \cdot \mathrm{OEt}_{2}$ was also performed. The results are illustrated in Scheme 10. 
<smiles>FC(F)(F)OC(OC(F)(F)F)(c1ccccc1)c1ccccc1</smiles>

BTI<smiles>Cc1ccccc1</smiles>

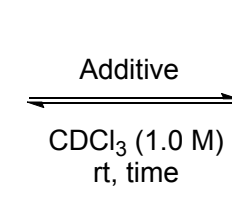

$1 \mathrm{~b}$<smiles>FC(F)(F)OC(OC(F)(F)F)(OC(F)(F)F)c1ccc(Br)cc1</smiles>

7



found

$\begin{array}{ll} & +1.2 \\ \text { calculated } & +0.6\end{array}$

\begin{tabular}{l|cc} 
Additive & time (h) & conv. (\%) \\
\hline none & 19 & 6 \\
& 41 & 13 \\
$\mathrm{BF}_{3} \cdot \mathrm{OEt}_{2}(5 \mathrm{~mol} \%)$ & 19 & 25 \\
& 41 & 26
\end{tabular}<smiles>Ic1ccccc1</smiles>

(kcal/mol)

Scheme 10. Evaluation of BTI toward uncatalyzed and catalyzed iodane metathesis.

The experimental outcome of the iodane metathesis reactions with BTI is in agreement with the calculation results. Metathesis occurs slowly in the absence of a Lewis acid; equilibrium could not be achieved, even after $41 \mathrm{~h}$. As with DIB, the Lewis acid has a drastic effect on the rate of metathesis, as equilibrium is attained within $19 \mathrm{~h}$. Again, the calculated free energy of reaction is in good agreement with the experimentally observed conversion (Scheme 10).

As the iodane metathesis reactions are under thermodynamic control, we selected a substrate that would favor a complete metathetical redox process. We performed the iodane metathesis using DIB and o-iodobenzoic acid (o-I-BzOH) as the iodoarene (Scheme 11). The thermodynamic drive (i.e., entropic gain) of the reaction was predicted to lead to a complete conversion $\left(\Delta \mathrm{G}_{\mathrm{rxn}}=-11.8 \mathrm{kcal} / \mathrm{mol}\right)$. Additionally, $o-\mathrm{I}-\mathrm{BzOH}$ enables the evaluation of the proximity effect of the iodine(I) and iodine(III) partners on the rate of the metathetical redox reaction. Indeed, $o-\mathrm{I}-\mathrm{BzOH}$ can serve as an acyloxy ligand. We thus performed $\mathrm{AcO} / o-\mathrm{I}-\mathrm{BzO}$ exchange on DIB in the absence of a Lewis acid. Treatment of an equimolar mixture of DIB and $o-\mathrm{I}-\mathrm{BzOH}$ in chloroform, followed by $\mathrm{AcOH}$ removal through hexane azeotrope, resulted in a clean mixture of DIB, 8, and $\mathbf{9}$ in a 1:2:1 ratio, indicative of an almost statistical equilibrium. It is important to note that no iodobenzene was observed at this point. The proximity effect of the I(I) and I(III) partners did not facilitate in any way the metathetical redox process in the absence of a Lewis acid. Treatment of this statistical mixture of [bis(acyloxy)iodo]benzenes with a catalytic quantity of $\mathrm{BF}_{3} \cdot \mathrm{OEt}_{2}$ resulted in the smooth and clean conversion to the single cyclic iodane $\mathbf{1 0}$ and iodobenzene. The same efficient metathetical redox process could be done in one-pot by treating an equimolar quantity of DIB and $o-\mathrm{I}-\mathrm{BzOH}$ with $5 \mathrm{~mol} \%$ of $\mathrm{BF}_{3} \cdot \mathrm{OEt}_{2}$ for $4 \mathrm{~h}$ in dichloromethane. Compound $\mathbf{1 0}$ could be obtained in essentially quantitative yield by simple removal of $\mathrm{PhI}$ by trituration with hexane.

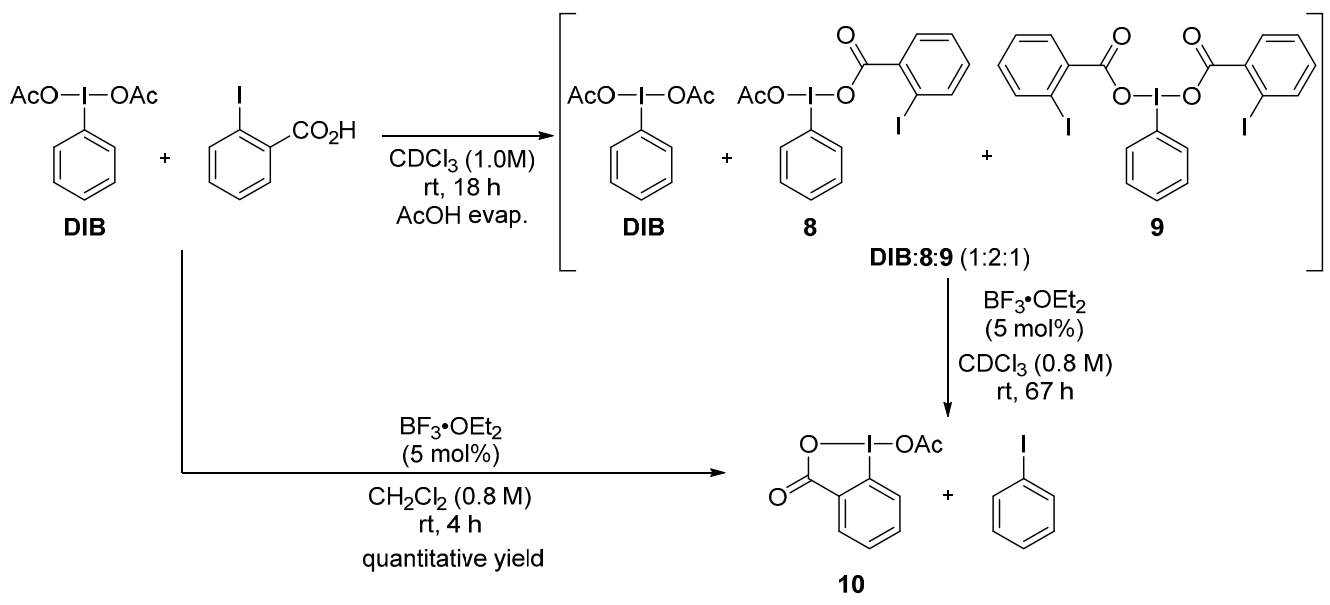

Scheme 11. Ligand exchange between DIB and $o-\mathrm{I}-\mathrm{BzOH}$ followed by iodane metathesis. 
There is a need to reconcile the fact that ligand exchange on DIB is facile at room temperature without the need for any activation, while the metathetical redox process is not possible. The nature of the exchange processes needs to be considered. While the calculations suggest a mandatory dissociative process through an iodonium intermediate to achieve metathesis, an associative mechanism could still be considered for ligand exchange (Scheme 12). It was calculated that cis-DIB, a T-shaped isomer of DIB in which both acetoxy ligands are cis to each other, would be energetically accessible at room temperature. This intermediate neutral iodane would react readily with a carboxylic acid ( $\mathrm{ACOH}$ was used for simplicity), through proton transfer, to afford in return DIB in its most stable conformation, bearing its two most electron withdrawing groups in trans relation. Addition of iodobenzene on cis-DIB cannot occur readily, as it is not a strong enough donor to stabilize expulsion of the acetoxy group.

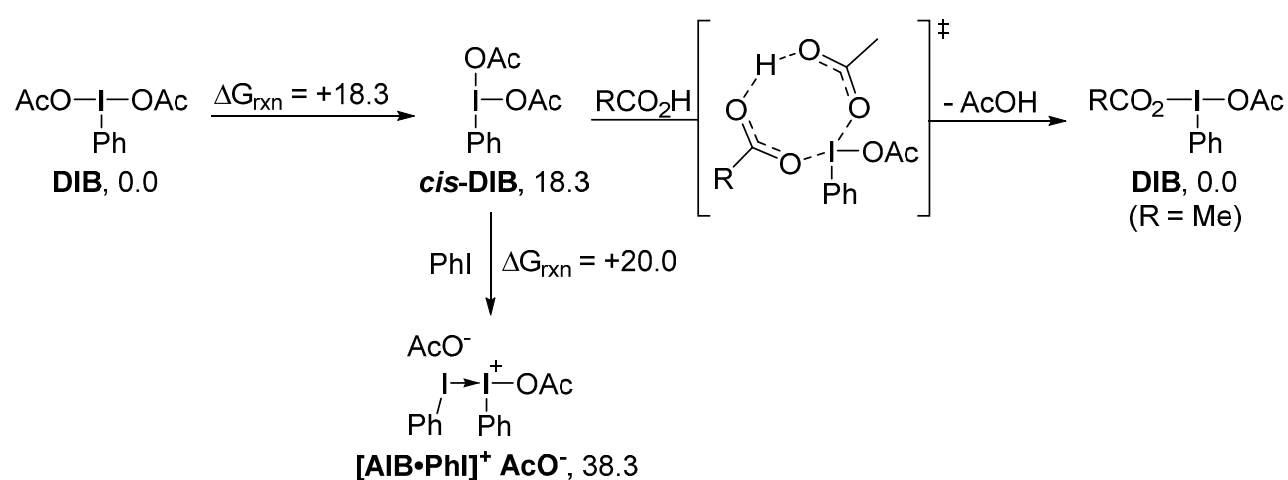

Scheme 12. Plausible mechanism for ligand exchange (energies reported in $\mathrm{kcal} / \mathrm{mol}$ ).

In summary, we have demonstrated that, with the simple action of a Lewis acid, it is possible to achieve the metathetical redox reaction of (diacetoxyiodo)arenes and iodoarenes. Both the experimental results and computational insights draw a clearer picture of what is needed for these metathetical redox reactions to proceed. The results presented herein open the way for further exploration.

\section{Experimental Section}

\subsection{General Information}

All glassware was stored in the oven and/or was flame dried prior to use under an inert atmosphere of gas. $\mathrm{CDCl}_{3}$ was dried over anhydrous $\mathrm{K}_{2} \mathrm{CO}_{3}$ but not thoroughly dried. Analytical thin-layer chromatography (TLC) was performed on precoated, glass-backed silica gel (Merck $60 \mathrm{~F}_{254}$ ). Visualization of the developed chromatogram was performed by UV absorbance, aqueous cerium molybdate, ethanolic phosphomolybdic acid, iodine, or aqueous potassium permanganate. Nuclear magnetic resonance spectra $\left({ }^{1} \mathrm{H},{ }^{13} \mathrm{C}, \mathrm{DEPT}\right)$ were recorded either on an Avance III HD 300 (Bruker, Billerica, MA, USA) or Mercury+ 400 (Agilent Technology, Santa Clara, CA, USA) spectrometers. Chemical shifts for ${ }^{1} \mathrm{H}-\mathrm{NMR}$ spectra are recorded in parts per million from tetramethylsilane with the solvent resonance as the internal standard. Data are reported as follows: chemical shift, multiplicity $(\mathrm{s}=$ singlet, $\mathrm{d}=$ doublet, $\mathrm{t}=$ triplet, $\mathrm{q}=$ quartet, $\mathrm{qn}=$ quintet, sext $=$ sextuplet, $\mathrm{m}=$ multiplet and $\mathrm{br}=$ broad), coupling constant in $\mathrm{Hz}$, integration. Chemical shifts for ${ }^{13} \mathrm{C}$-NMR spectra are recorded in parts per million from tetramethylsilane with the solvent resonance as the internal standard. All spectra were obtained with complete proton decoupling. When ambiguous, proton and carbon assignments were established using COSY, NOESY, HMQC and DEPT experiments. High resolution mass spectra were recorded on a Maxis ESI-Q-Tof (Bruker, Billerica, MA, USA) at the Université de Sherbrooke. 


\subsection{Metathetical Redox Reaction of HTIB and p-Methyliodobenzene}

To a round-bottom flask were added [hydroxy(tosyloxy)iodo]benzene (HTIB) (333.4 mg, $0.850 \mathrm{mmol}), p$-methyliodobenzene $(185.3 \mathrm{mg}, 0.850 \mathrm{mmol})$ and dichloromethane $(1.7 \mathrm{~mL}, 0.5 \mathrm{M})$. The white suspension was stirred at room temperature. Aliquots $(5-10 \mu \mathrm{L})$ were taken from clear dichloromethane supernatant after allowing the suspension to settle and diluted in HPLC-grade hexanes $(500-750 \mu \mathrm{L})$ at various moments. Conversions were determined by GC-MS by integration of signals of iodobenzene and $p$-methyliodobenzene.

\subsection{Lewis-Acid-Catalyzed Metathetical Redox Reaction of DIB and p-Bromoiodobenzene}

To a flame-dried vial equipped with a septum, under an argon atmosphere, were added DIB $(372.1 \mathrm{mg}, 1.155 \mathrm{mmol}), p$-bromoiodobenzene $(325.6 \mathrm{mg}, 1.155 \mathrm{mmol})$ and $\mathrm{CDCl}_{3}(442 \mu \mathrm{L})$.

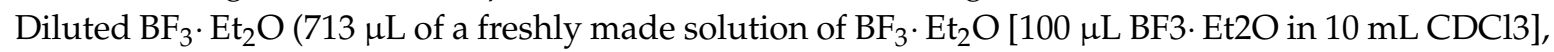
$0.058 \mathrm{mmol}$ ) was added for a total volume of $1.16 \mathrm{~mL}$ of $\mathrm{CDCl}_{3}(1 \mathrm{M})$. The solution was stirred at room temperature under inert atmosphere. Conversions were determined by integration of well-defined ${ }^{1} \mathrm{H}-\mathrm{NMR}$ signals ( $5 \mathrm{~s}$ relaxation time) of the two (diacetoxyiodo)arenes present in solution. The free energies of reaction (Equation (1) and Scheme 10) were determined with the measured conversions (see Supplementary Materials for more details).

\subsection{One-Pot Metathetical Redox Reaction of DIB and o-Iodobenzoic Acid}

To a flame-dried vial, under argon, were added o-iodobenzoic acid $(247.7 \mathrm{mg}, 1.00 \mathrm{mmol})$, DIB (322.1 mg, $1.00 \mathrm{mmol}$ ) and a solution of $\mathrm{BF}_{3} \cdot \mathrm{Et}_{2} \mathrm{O}\left(1.22 \mathrm{~mL}\right.$, [100 $\mu \mathrm{LF}_{3} \cdot \mathrm{Et}_{2} \mathrm{O}$ in $\left.10 \mathrm{~mL} \mathrm{CH}_{2} \mathrm{Cl}_{2}\right]$, $0.012 \mathrm{mmol}$ ). The final reaction concentration is $0.8 \mathrm{M}$. The solution was allowed to stir at room temperature for $4 \mathrm{~h}$. The solvent was evaporated and the crude residue washed twice with hexane. The crude product was dried under vacuum to afford compound 10 in quantitative yield. The spectral data is consistent with the reported one in the literature [26].

\section{Computational Details}

The geometry optimizations were done using the Gaussian 09 software package [27] with the M06-2X [28] density functional in combination with the 6-31+G(d,p) basis set $[29,30]$ for all atoms except iodine, for which LANL2DZdp + ECP was used [31,32]. The structures were optimized with a solvation model (SMD) for chloroform [33]. Unless otherwise stated, a fine grid density was used for numerical integration in the calculations. Conformational searches were done on all the species described throughout the mechanistic pathways. Harmonic vibrational frequencies were computed for all optimized structures to verify that they were either minima or transition states, possessing zero or one imaginary frequency, respectively. All the free energies are reported in $\mathrm{kcal} / \mathrm{mol}$ and incorporate unscaled free energy corrections based on the vibrational analyses and temperature of $298 \mathrm{~K}$.

Supplementary Materials: Supplementary materials can be accessed at: http:/ /www.mdpi.com/1420-3049/20 / 12/19874/s1. Cartesian coordinates, electronic and zero-point vibrational energies.

Acknowledgments: This work was supported by the National Science and Engineering Research Council (NSERC) of Canada, the Canada Foundation for Innovation (CFI), the FRQNT Centre in Green Chemistry and Catalysis (CGCC), and the Université de Sherbrooke.

Author Contributions: A.J.-D.L. and C.Y.L. conceived and designed the experiments; A.J.-D.L. performed the experiments; C.Y.L. performed the computational calculations; C.Y.L. wrote the paper; A.J.-D.L. helped with the correction of the paper.

Conflicts of Interest: The authors declare no conflict of interest.

\section{References}

1. Zhdankin, V.V. Hypervalent. Iodine Chemistry; John Wiley \& Sons: Chichester, UK, 2014. 
2. Wirth, T. Hypervalent. Iodine Chemistry; Springer: Berlin, Germany, 2003.

3. Moriarty, R.M.; Prakash, O. Oxidation of phenolic compounds with organohypervalent iodine reagents. Org. React. 2001, 57, 327-415.

4. Varvoglis, A. Hypervalent. Iodine in Organic Synthesis; Academic Press: San Diego, CA, USA, 1997.

5. Varvoglis, A. The Organic Chemistry of Polycoordinated Iodine; VCH: New York, NY, USA, 1992.

6. Uyanik, M.; Ishihara, K. Hypervalent iodine-mediated oxidation of alcohols. Chem. Commun. 2009, 16, 2086-2099. [CrossRef] [PubMed]

7. Zhdankin, V.V.; Stang, P.J. Chemistry of polyvalent iodine. Chem. Rev. 2008, 108, 5299-5358. [CrossRef] [PubMed]

8. Wirth, T. Hypervalent iodine chemistry in synthesis: Scope and new directions. Angew. Chem. Int. Ed. 2005, 44, 3656-3665. [CrossRef] [PubMed]

9. Moriarty, R.M. Organohypervalent iodine: Development, applications, and future directions. J. Org. Chem. 2005, 70, 2893-2903. [CrossRef] [PubMed]

10. Zhdankin, V.V.; Stang, P.J. Recent developments in the chemistry of polyvalent Iodine compounds. Chem. Rev. 2002, 102, 2523-2574. [CrossRef] [PubMed]

11. Zhdankin, V.V. Hypervalent iodine(III) reagents in organic synthesis. ARKIVOC 2009, 2009, 1-62.

12. Varvoglis, A. Aryliodine(III) dicarboxylates. Chem. Soc. Rev. 1981, 10, 377-407. [CrossRef]

13. Yusubov, M.S.; Wirth, T. Solvent-free reactions with hypervalent iodine reagents. Org. Lett. 2005, 7, 519-521. [CrossRef] [PubMed]

14. Yamamoto, Y.; Togo, H. Facile One-pot preparation of [hydroxy(sulfonyloxy)iodo]arenes from iodoarenes with MCPBA in the Presence of Sulfonic Acids. Synlett 2005, 2486-2488. [CrossRef]

15. Sharefkin, J.G.; Saltzman, H. Iodosobenzene diacetate. Org. Synth. 1963, 43, 62.

16. Koser, G.F.; Wettach, R.H. [Hydroxy(tosyloxy)iodo]benzene, a versatile reagent for the mild oxidation of aryl iodides at the iodine atom by ligand transfer. J. Org. Chem. 1980, 45, 1542-1543. [CrossRef]

17. Rabah, G.A.; Koser, G.F. Facile synthetic entry into the 1,3-dihydro-3-methyl-3-phenyl-1,2-benziodoxole family of $\lambda^{3}$-iodanes. Tetrahedron Lett. 1996, 37, 6453-6456. [CrossRef]

18. Geary, G.C.; Hope, E.G.; Singh, K.; Stuart, A.M. Electrophilic fluorination using a hypervalent iodine reagent derived from fluoride. Chem. Commun. 2013, 49, 9263-9265. [CrossRef] [PubMed]

19. Kasahara, T.; Jang, Y.J.; Racicot, L.; Panagopoulos, D.; Liang, S.H.; Ciufolini, M.A. Iodonium metathesis reactions. Angew. Chem. Int. Ed. 2014, 53, 9637-9639. [CrossRef] [PubMed]

20. Beaulieu, S.; Legault, C.Y. Mechanistic insights on the iodine(III)-mediated $\alpha$-oxidation of ketones. Chem. Eur. J. 2015, 21, 11206-11211. [CrossRef] [PubMed]

21. Koser, G.F. [Hydroxy(tosyloxy)iodo]benzene and closely related iodanes: The second stage of development. Aldrichimica Acta 2001, 34, 89-102. [CrossRef]

22. Richter, H.W.; Cherry, B.R.; Zook, T.D.; Koser, G.F. Characterization of species present in aqueous solutions of [hydroxy(mesyloxy)iodo]benzene and [hydroxy(tosyloxy)iodo]benzene. J. Am. Chem. Soc. 1997, 119, 9614-9623. [CrossRef]

23. Ochiai, M.; Sueda, T.; Miyamoto, K.; Kiprof, P.; Zhdankin, V.V. Trans Influences on hypervalent bonding of aryl $\lambda^{3}$-iodanes: Their stabilities and isodesmic reactions of benziodoxolones and benziodazolones. Angew. Chem. Int. Ed. 2006, 118, 8383-8386. [CrossRef]

24. Zhong, W.; Yang, J.; Meng, X.; Li, Z. $\mathrm{BF}_{3} \cdot \mathrm{OEt}_{2}$-Promoted diastereoselective diacetoxylation of alkenes by $\mathrm{PhI}(\mathrm{OAc})_{2}$. J. Org. Chem. 2011, 76, 9997-10004. [CrossRef] [PubMed]

25. Ochiai, M.; Takeuchi, Y.; Katayama, T.; Sueda, T.; Miyamoto, K. Iodobenzene-catalyzed $\alpha$-acetoxylation of ketones. In situ generation of hypervalent (diacyloxyiodo)benzenes using m-chloroperbenzoic acid. J. Am. Chem. Soc. 2005, 127, 12244-12245. [CrossRef] [PubMed]

26. Waser, J.; Vita, M.V. Azidation of $\beta$-keto esters and silyl enol ethers with a benziodoxole reagent. Org. Lett. 2013, 15, 3246-3249.

27. Frisch, M.J.; Trucks, G.W.; Schlegel, H.B.; Scuseria, G.E.; Robb, M.A.; Cheeseman, J.R.; Scalmani, G.; Barone, V.; Mennucci, B.; Petersson, G.A.; et al. Gaussian 09, Revision C.01. Gaussian, Inc.: Wallingford, CT, USA, 2009. 
28. Zhao, Y.; Truhlar, D.G. The M06 suite of density functionals for main group thermochemistry, thermochemical kinetics, noncovalent interactions, excited states, and transition elements: Two new functionals and systematic testing of four M06-class functionals and 12 other functionals. Theor. Chem. Acc. 2008, 120, 215-241.

29. Hehre, W.J.; Ditchfield, R.; Pople, J.A. Self-consistent molecular orbital methods. XII. Further extensions of gaussian-type basis sets for use in molecular orbital studies of organic molecules. J. Chem. Phys. 1972, 56, 2257-2261. [CrossRef]

30. Hariharan, P.C.; Pople, J.A. The influence of polarization functions on molecular orbital hydrogenation energies. Theor. Chim. Acta 1973, 28, 213-222. [CrossRef]

31. Wadt, W.R.; Hay, P.J. Ab initio effective core potentials for molecular calculations. Potentials for main group elements Na to Bi. J. Chem. Phys. 1985, 82, 284-298. [CrossRef]

32. Check, C.E.; Faust, T.O.; Bailey, J.M.; Wright, B.J.; Gilbert, T.M.; Sunderlin, L.S. Addition of polarization and diffuse functions to the LANL2DZ basis set for P-block elements. J. Phys. Chem. A 2001, 105, 8111-8116. [CrossRef]

33. Marenich, A.V.; Cramer, C.J.; Truhlar, D.G. Universal solvation model based on solute electron density and on a continuum model of the solvent defined by the bulk dielectric constant and atomic surface tensions. J. Phys. Chem. B 2009, 113, 6378-6396. [CrossRef] [PubMed]

Sample Availability: Not available.

(C) 2015 by the authors; licensee MDPI, Basel, Switzerland. This article is an open access article distributed under the terms and conditions of the Creative Commons by Attribution (CC-BY) license (http://creativecommons.org/licenses/by/4.0/). 